\title{
3d Monte Carlo analysis on photons step through turbid medium by Mie scattering
}

\author{
E. E. Pérez Mayesffer \\ Facultad de Ciencias Físico Matemáticas, Benemérita Universidad Autónoma de Puebla, \\ Av. San Claudio s/n Col. San Manuel 72570 Puebla Puebla. \\ e-mail: emayesffer@gmail.com \\ E. Reynoso Lara \\ Facultad de Ciencias de la Electrónica, Benemérita Universidad Autónoma de Puebla, \\ Av. San Claudio s/n Col. San Manuel 72570 Puebla Puebla. \\ W. F. Guerrero Sánchez \\ Facultad de Ciencias Físico Matemáticas Benemérita Universidad Autónoma de Puebla, \\ Av. San Claudio s/n Col. San Manuel 72570 Puebla Puebla. \\ G. Rodríguez Zurita QPD \\ Facultad de Ciencias Físico Matemáticas Benemérita Universidad Autónoma de Puebla, \\ Av. San Claudio s/n Col. San Manuel 72570 Puebla Puebla. \\ J. Dávila Pintle \\ Facultad de Ciencias de la Electrónica, Benemérita Universidad Autónoma de Puebla, \\ Av. San Claudio s/n Col. San Manuel 72570 Puebla Puebla. \\ Y. E. Bravo-García \\ Facultad de Ciencias de la Electrónica, Benemérita Universidad Autónoma de Puebla, \\ Av. San Claudio s/n Col. San Manuel 72570 Puebla Puebla.
}

Received 8 September 2020; accepted 1 December 2020

\begin{abstract}
Photon scattering profiles in a turbid media were investigated through numerical simulation based on the Monte Carlo-Mie method at this present work, using Wolfram Mathematics in the program algorithm. Photon scattering was treated using electromagnetic spherical harmonics waves in three-dimensional scattering. The proposal, as an alternative to the Henyey-Greenstein phase approximation, was defining a unit vector that represents a phase distribution, as an equivalent function with three vector components, within the turbid media. Associating the step component, as projection using Legendre polynomials and for the transverse plane components were defining as vector bases in terms of Legendre-Hankel functions, according to Gustav Mie's theory. This composite vector was defined as a step function and was evaluated within the Monte Carlo algorithm, obtaining simulations of light scattering. Backscatter profiles were compared for different geometric dimensions of the spherical particles within the turbid media, including validation of the model with an experimental Lidar signal from low clouds, obtaining physical properties of the turbid media by the proposed theoretical model.
\end{abstract}

Keywords: Photon scattering; turbid media; Monte Carlo-Mie method; spherical harmonics.

PACS: $02.05 . \mathrm{Ng} ;$ 42.25.Fx; 42.68.Mj

DOI: https://doi.org/10.31349/RevMexFis.67.292

\section{Introduction}

A scientist community that involves in scattering light studies, today they offer to present many scattering light models, increasingly developed in order to obtain and identify, through the study of properties, some different atmospheric elements of our planet and outer space. This is an important contribution of these scattering light models about climate change and environmental sustainability to the planet. Nowdays in Mexico, there are not many registers of scattering light works by research groups; that is why this work presents the development and testing of one combined Monte Carlo-
Mie model, being careful with the focus on special functions of scatterer unit vector from the proposed work. This work was successful thanks to the actual current computer tools.

Some works reported by researchers as L.R. Bissonnette, et al., Wu Zhensen et al., P. F. Liaparinos, and Yuzaho Ma et al. use within their modeling an approximate simplified phase function called the Henyey-Greenstein function. It is applied to scatterer particles with spherical symmetry, [1-4]. In this work, the authors have developed a physical model in one simplified algorithm considering a completely analytical dominium and testings to get theoretical results according to spherical harmonics and Lidar theory. 


\section{Development of the Monte Carlo Mie model}

This section is a review of spherical harmonics for scattered waves. Emphasis was being placed on handling special functions that make up the electromagnetic fields with spherical symmetry.

In the second part, the principal concepts are analyzed about handling the statistic-numerical Monte Carlo method. Finally, the purpose of fuse both of them in one combined model is exposed.

\subsection{Amplitude of scattering waves}

The outer/inner relation of the electromagnetic fields, from the interaction of electromagnetic radiation with a spherical scatterer of radius a, is defined as

$\left(\mathbf{E}_{i n}+\mathbf{E}_{s c}-\mathbf{E}_{i}\right) \times \hat{\boldsymbol{e}_{r}}=\left(\mathbf{H}_{i n}+\mathbf{H}_{s c}-\mathbf{H}_{i}\right) \times \hat{\boldsymbol{e}_{r}}=0$,

Defining as: $\mathbf{E}_{\text {in }}$ incident electric field, $\mathbf{H}_{\text {in }}$ incident magnetic field, $\mathbf{E}_{i}$ inner electric field, $\mathbf{H}_{i}$ inner magnetic field, $\mathbf{E}_{\mathrm{sc}}$ scattering electric field, and $\mathbf{H}_{s} \mathrm{c}$ scattering magnetic field. These fields were proposed by Mie [5]. For this work the most important fields are the scattered fields, these are written as Hankel special functions, which convergence at infinity.

A set of equations resulting from boundary conditions of Eq. (1), solving these equations for the scattered fields and finish getting the Mie Coefficients $\left(a_{n}, b_{n}\right)$, which are related with the amplitude of scattering waves as:

$$
\begin{aligned}
a_{n} & =\frac{m \psi_{n}(m x) \psi_{n}^{\prime}(x)-\psi_{n}(x) \psi_{n}^{\prime}(m x)}{m \psi_{n}(m x) \xi_{n^{\prime}}(x)-\xi_{n}(x) \psi_{n}^{\prime}(m x)^{\prime}}, \\
b_{n} & =\frac{\psi_{n}(m x) \psi_{n}^{\prime}(x)-m \psi_{n}(x) \psi_{n}^{\prime}(m x)}{\psi_{n}(m x) \xi_{n^{\prime}}(x)-m \xi_{n}(x) \psi_{n}^{\prime}(m x)} .
\end{aligned}
$$

As Mie's theory establishes, the scattering electric field can be possible to define by two orthogonal vector components $\left(E_{\mathrm{sc}_{\theta}}, E_{\mathrm{sc}_{\phi}}\right)$ to produce a transverse plane, which is perpendicular to the scattering direction. From the definition of scalar scattering electric field, it is obtained [5]

$$
E_{\mathrm{sc}_{\theta}}=E_{\mathrm{sc}_{\theta}}+E_{\mathrm{sc}_{\phi}}
$$

and

$$
\begin{aligned}
& E_{\mathrm{sc}_{\theta}}=\frac{\cos \phi}{\rho} \sum_{n=1}^{\infty} E_{n}\left(i a_{n} \xi_{n}^{\prime} \tau_{n}-b_{n} \xi_{n} \pi_{n}\right), \\
& E_{\mathrm{sc}_{\phi}}=\frac{\cos \phi}{\rho} \sum_{n=1}^{\infty} E_{n}\left(b_{n} \xi_{n} \tau_{n}-i a_{n} \xi_{n}^{\prime} \pi_{n}\right) \cdot \Delta
\end{aligned}
$$

Noting that $\rho=k r, k$ is the wavenumber and $r$ is the distance of the scattering wave from the scatterer.

Applying the optical theorem that defines one transversal section of extinction $(\sigma)$ as the relation between the extinct total energy $W_{\text {total }}$ (sum of absorption and scattering energies) and the incident irradiance [5]. It is possible to get one transversal scattering section as,

$$
\begin{aligned}
\sigma & =\frac{4 \pi}{k^{2}} \mathbf{R e}\left\{S\left(0^{0}\right)\right\}=\frac{2 \pi}{k^{2}} \\
& \times\left\{\sum_{n=1}^{\infty}(2 n+1) \mathbf{R e}\left(a_{n}+b_{n}\right)\right\},
\end{aligned}
$$

normalizing Eq. (7) to one geometric area, then

$$
\frac{\sigma}{\pi(a)^{2}}=\frac{2}{(k a)^{2}}\left\{\sum_{n=1}^{\infty}(2 n+1) \mathbf{R e}\left(a_{n}+b_{n}\right)\right\} .
$$

To get the scattering transversal section $\sigma_{s}$, it is necessarily integrated the total scattering power over a solid angle $4 \pi$, as [6]

$$
\sigma_{s}=\frac{2 \pi}{k^{2}} \int_{0}^{\pi} \operatorname{sen} \theta d \theta\left(\left|S_{1}(\theta)\right|^{2}+\left|S_{2}(\theta)\right|^{2}\right),
$$

normalizing Eq. (9) with one geometric area, then

$$
\frac{\sigma_{s}}{\pi(a)^{2}}=\frac{2}{(k a)^{2}} \sum_{n=1}^{\infty}(2 n+1)\left(\left|a_{n}\right|^{2}+\left|b_{n}\right|^{2}\right)
$$

Finally, by energy conservation, it is possible to get,

$$
\sigma=\sigma_{s}+\sigma_{c} .
$$

In other words, the transversal section of extinction from one scatterer particle, it is conforming by the sum of the transversal section of scattering $\sigma_{s}$ and the transversal section of absorption $\sigma_{c}$.

The volumetric coefficient of extinction in the turbid media is defined by,

$$
\beta=N(z) \sigma,
$$

Noting that, $N(z)$ is the normalized concentration of particles, from turbid media, in one illuminated unit volume by one incident pulse of light.

\subsection{Monte Carlo Method}

One synthetized explanation of the terms involved in the simulation in relation to a distribution of particles inside of turbid medium, by Monte Carlo then is given.

Suppose a representation of one turbid medium with a hypotetical homogeneous thick $h(0<x<h)$ and walls of infinite areas where $\omega_{k}$ photons from a gaussian pulse of energy are impacted.

Considering Eqs. (8), (10) and (11), there is the probability $\left(\sigma_{s} / \sigma\right)$, that an average of photons is in scattering process and the weightening them being defined by [7],

$$
\omega_{k}\left(\sigma_{s} / \sigma\right)
$$

there is also the probability $\left(\sigma_{c} / \sigma\right)$ that an average of photons is in absorption process and the weightening them being defined by,

$$
\omega_{k}\left(\sigma_{c} / \sigma\right) .
$$


The free path $(\lambda)$, between the present and the next photon train scattering event, is proposed with an exponential distribution density $(\gamma)$, and is defined by the equation,

$$
\lambda=-\frac{1}{\sigma} \ln (\gamma)
$$

Calculating the abscissa at the next collision point, results

$$
x_{k+1}=x_{k}+\lambda_{k} \mu_{k},
$$

with $\mu_{k}=\cos \theta$. It represents the new direction of the rest of the train, once it has already collided.

\subsection{Monte Carlo-Mie model}

Noting explanation from Secs. 2.1 and 2.2. Based on those observations, the following is proposed:

1.- Associate vector bases of the Mie model with the step function of the classic Monte Carlo model (Eq. (16)), generating a new unit vector of spatial advance and projected as spherical harmonics. Where the radial component has been projected from one polar axis, inside of turbid media, in this case it is not used the projection function $\mu_{k}=\cos \theta$. Instead can be proposed one spherical harmonic function as Legendre spatial functions, choosing one order $m=1$ to ensure azimuthal symmetry $[5,8]$ as

$$
\mu_{k 1}=P_{n}^{1}\left(\cos \theta_{k}\right)
$$

Now for the second and third components from unit vector, they are proposed as vector components from Mie scattering electric field: parallel component $E_{\| s}\left[\mathbf{N}_{P 1 n}^{(3)}\right]$ and perpendicular component $E_{\perp s}\left[\mathbf{M}_{I 1 n}^{(3)}\right]$.

Where the parallel component $E_{\| s}$ is linked with the unit vector $\hat{e}_{\theta}$, taking the scalar component as

$$
\mu_{k 2}=\left\{i \tau \xi_{n}^{\prime}-\pi_{n} \xi_{n}\right\} \frac{\cos \psi}{\rho}
$$

the perpendicular component $E_{\perp s}$ is linked with $\hat{e}_{\varphi}$, taking the scalar component as

$$
\mu_{k 3}=\left\{i \pi_{n} \xi_{n}^{\prime}-\tau_{n} \xi_{n}\right\} \frac{\sin \psi}{\rho} .
$$

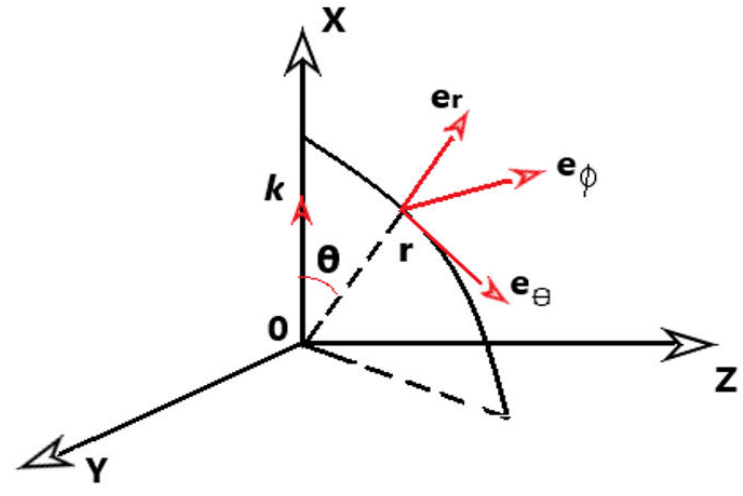

FIGURE 1. Reference system of $\mathbf{r}$ vector to $\mathbf{k}$ vector, expanding in spherical harmonics.

The complete expression of one unit vector, expanded on spherical harmonics, is

$$
\begin{aligned}
v & =\hat{e}_{r} r_{k+1}+\hat{e}_{\theta} \theta_{k+1}+\hat{e}_{\varphi} \varphi_{k+1}=\hat{e}_{r}\left(r_{k}+\lambda_{k} \mu_{k 1}\right. \\
& +\hat{e}_{\theta}\left(\theta_{k}+\lambda_{k r a d} \mu_{k 2}\right)+\hat{e}_{\varphi}\left(\psi_{k}+\lambda_{k r a d} \mu_{k 3}\right) .
\end{aligned}
$$

With the distribution function of free path between different scattering events, inside the medium, as

$$
\lambda_{k}=-\frac{1}{\sigma_{k}} \ln (\gamma)
$$

And the distribution function of free angular directions, as

$$
\lambda_{k r a d}=-\frac{1}{\sigma_{k r a d}} \ln \left(\gamma_{r a d}\right)
$$

One graphic representation in the space, is shown in the Fig. 1.

The vector proposed at Eq. (20) is similar to the proposed by Badrinath et al., [9]. The difference in this work was that the authors have proposed a projection in spherical harmonics.

2.- The amplitudes of scattering waves, associated to Mie coefficients (Eqs. (2) and (3)) are linked to the scattering and absorption probabilities inside turbid media.

3.- The incident theoretical light pulse proposed must has a gaussian shape to be closer a real light pulse.

\section{Algorithm flow chart}

Algorithm flow chart is composed by three blocks, which are:

Block 1. It is declared: turbid medium thickness, the coefficients of: scattering, absorption and extinction; the probabilities of: scattering, absorption and extinction; the numbers of: scattering events and photons.

This block generate: 
a) The probability distribution function, is the list of values resulting from convolution between the probability of total energy and the gaussian probability function of the incident pulse.

b) The position photonic function, that identifies the position of photons train, while it goes through the turbid medium. The position photonic function chooses the average probability photon values from the scattering profile distribution list $[w C C(R, \lambda)]$ generated, according to the position photon train.

c) The scattering photonic function, that identifies the photons train leaving the turbid medium in the forward direction. The scattering photonic function chooses the average probability photon values from the scattering profile distribution list $[w C C(R, \lambda)]$ generated, according to the scattering position photon train. d) The backscattering photonic function, that identifies the photons train leaving the turbid medium in the backward direction. The backscattering photonic function chooses the average probability photon values from the scattering profile distribution list $[w C C(R, \lambda)]$ generated, according to the backscattering position photon train.

e) A 3D step function $v$, which it is composed by: $\lambda_{k}$ value, it means a exponential distribution function of free paths between scattering events, $\lambda_{k r a d}$ value, it means a exponential distribution function of free directions between scattering events, $\mu$ value, it means all random data with polar angles, $\psi$ value, it means all random data in relation to azimuthal angles.

Block 2. Execution of photon step function, which is defined as a state vector:

$$
v=\left\{\begin{array}{c}
\left\{\left(\lambda_{k}, \theta_{k}, \varphi_{k}\right)\right\}, \quad \text { if } \mu_{k 1}=1, \quad \psi=0 \\
\left\{\operatorname{Re}\left[\left(r_{k}+\lambda_{k} \mu_{k 1}\right)\right]\right\},\left\{\operatorname{Arg}\left[\left(\theta_{k}+\lambda_{k r a d} \mu_{k 2}\right)\right], \operatorname{Arg}\left[\left(\psi_{k}+\lambda_{k r a d} \mu_{k 3}\right)\right]\right\}, \quad \text { if } \quad \mu_{k 1} \neq 1, \quad \psi \neq 0
\end{array} .\right.
$$

This block generate a list of all random trajectories in function of the number of scattering events and the photons number declared $[7,10]$. It results in a list of three elements; first for position and two for directions $\left(\theta_{k}, \psi_{k}\right)$. From the list $[w C C(R, \lambda)]$ generated, can be obtain another new list $\left[w C C(R, \lambda)_{F O V}\right]$ that includes the average probability photon values that are trapped inside the Field Of View (FOV) of a virtual photodetector, located at $(-R, 0,0)$ position, as is shown in Fig. 2.

The list $w C C(R, \lambda)_{\mathrm{FOV}}$ is the photon backscattering average probability located within the field of view of the telescope (FOV) whose value variation depends on the wavelength and also depends of the convergent exponential distribution function.

The algorithm of photon step through turbid media is shown in Fig. 3,

Block 3. Photons are counted and averaged in the photodetector. Besides they are shown in the screen editor as position profiles graphic.

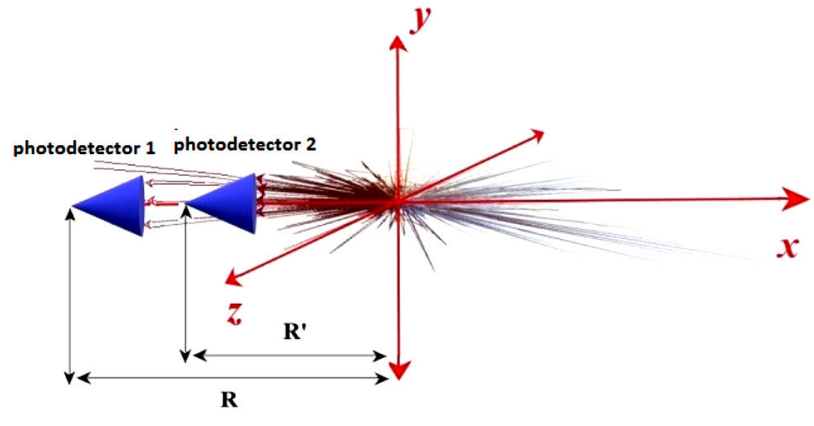

FIGURE 2. Backscatterig detection at $(-R 00)$ direction.
The calculus of the average probability photon values inside the photodetector was possible because of the complex vector components definition of the transverse plane, that is generated by the photon step function. The complex definition of parallel component, is

$$
\frac{\cos \phi}{\rho}\left\{i \tau_{n} \xi_{n}^{\prime}+\pi_{n} \xi_{n}\right\} \hat{e}_{\theta},
$$

and perpendicular component, is

$$
\frac{\sin \phi}{\rho}\left\{i \pi_{n} \xi_{n}^{\prime}+\tau_{n} \xi_{n}\right\} \hat{e}_{\varphi}
$$

As a result of this, the authors propose associate a couple of ortogonal semicomplex planes, and from that it could be possible to get measures of angular values in relation to the polar and azimuthal directions, at the moment of photons backscattering. The average probability photon values caught is depending of a solid angle from photodetector (FOV), which has semiangle of

$$
\boldsymbol{\beta}=\tan ^{-1} \frac{y}{R} .
$$

Equation (26) has a direct relation with the photodetector light acceptance cone (aperture of telescope). The numerator $y$, represent one aperture radius of telescope and the denominator $R$, represent the distance of photodetector to the turbid medium border. Another perspective is shown in the Fig. 4.

\section{Results of simulations}

It is made simulations, considering a turbid media not absorbent, with the following parameters: an incident light 


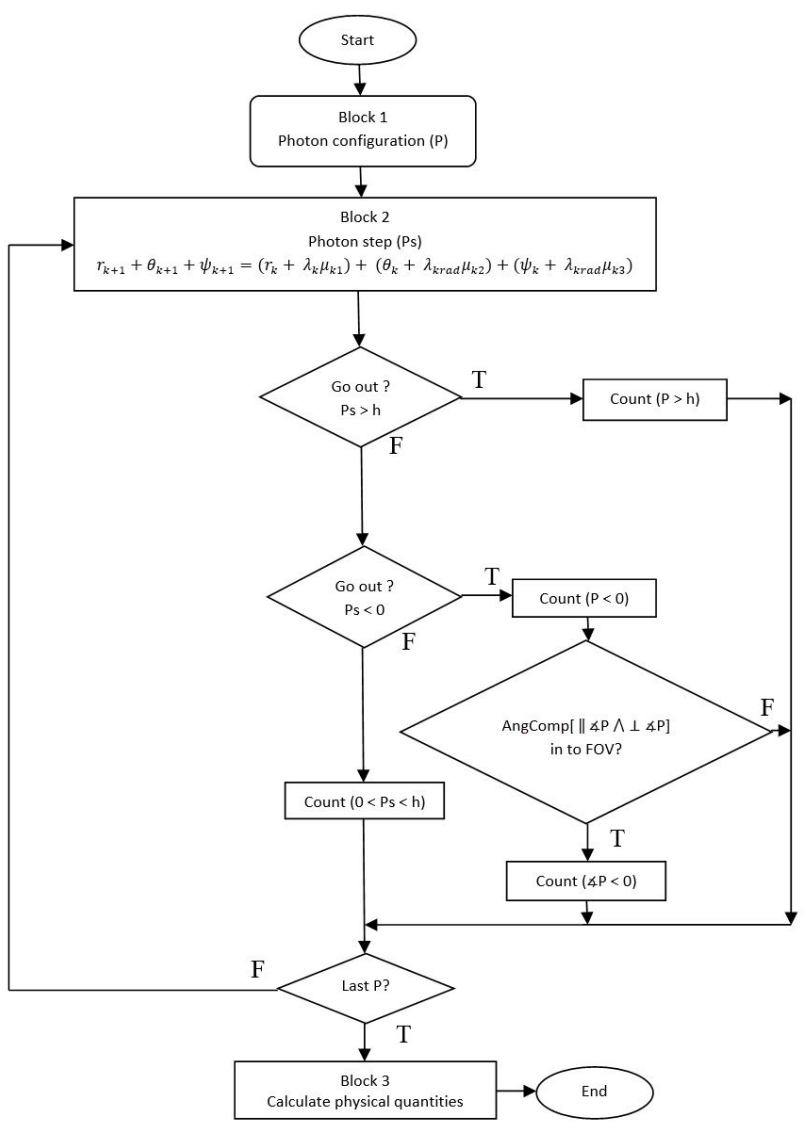

FIGURE 3. Flow chart.

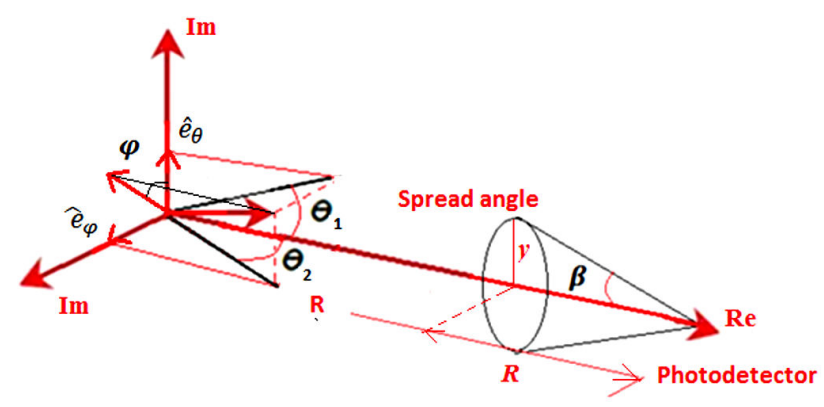

FIGURE 4. Backscattering inside of field of view (FOV).

pulse composed by population of $5.5 \times 10^{5}$ photons, 100 scattering events, $532 \mathrm{~nm}$ of wavelength, $h=2 \mathrm{~m}$ thickness of turbid media, $r=8.65 \mu \mathrm{m}, r=10 \mu \mathrm{m} \mathrm{y} r=12.5 \mu \mathrm{m}$ radius dimension of scatterers and one refractive index of 1.33.

\subsection{Comparing average result and space distributions}

Tables I, II, III shown the average of: backscattered photons probability, transmitted photons probability and caught photon probability through out one turbid media composed by spherical scatterers. Plotting their spatial profiles of each of them.

Noting that when the physical parameters as: scatterer radius and refractive index are used into the scattering coefficient and absorption coefficient and considering the Mie
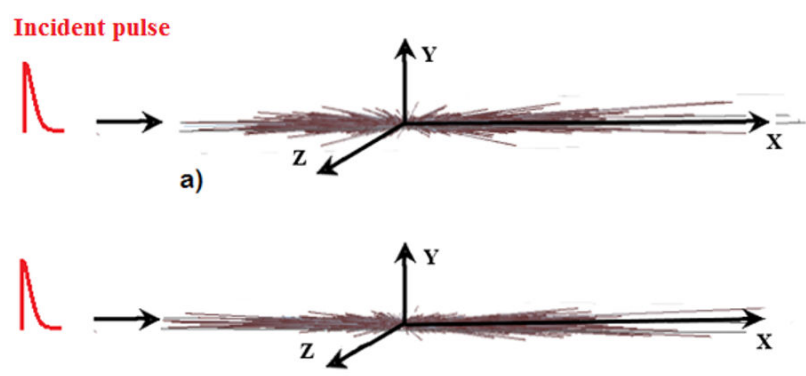

b)

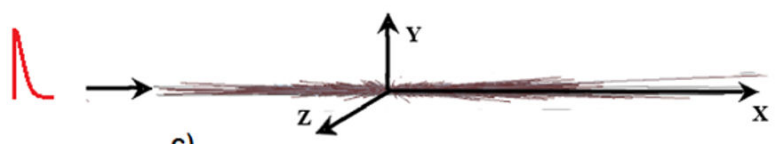

c)

FIGURE 5. Spatial distribution profiles, from droplets water turbid media of $2 \mathrm{~m}$ of thickness, with relative refractive index of $n=1.33$ and radius of: a) $r=8.65 \mu \mathrm{m}$, b) $r=10 \mu \mathrm{m}$ and c) $r=12.5 \mu \mathrm{m}$.

vector bases into state function. Scattering light is observed with an increasingly forward direction (toward direction of incident pulse) and less in backward direction, it happen every time that scatterers size is increasing. The last concept is checked with the results of the photon concentrations in forward, backward and trapped cases, given by the program in relation to turbid media as it is shown in Tables I, II and III. Also, the scattering spatial distribution is similar to the frontal lobe of radiation from the Mie phase function [5].

\subsection{Comparison of backscattered photons as a func- tions of distance}

The profile of distance distributions of backscattered photons probability is calculated from 5 to $50 \mathrm{~m}$, by steps of $5 \mathrm{~m}$. Figure 6 , shows the profile of average probability photon values versus distance. For $r=8.65,10$ and $12.5 \mu \mathrm{m}$ of scatterer.

Comparing $5.5 \times 10^{5}$ of photons hitting the turbid media (Fig. 6), from each distance, the exponential distribution profiles again shows a strong dependence of the size of the scatterer in relation with backscattering photons probability.

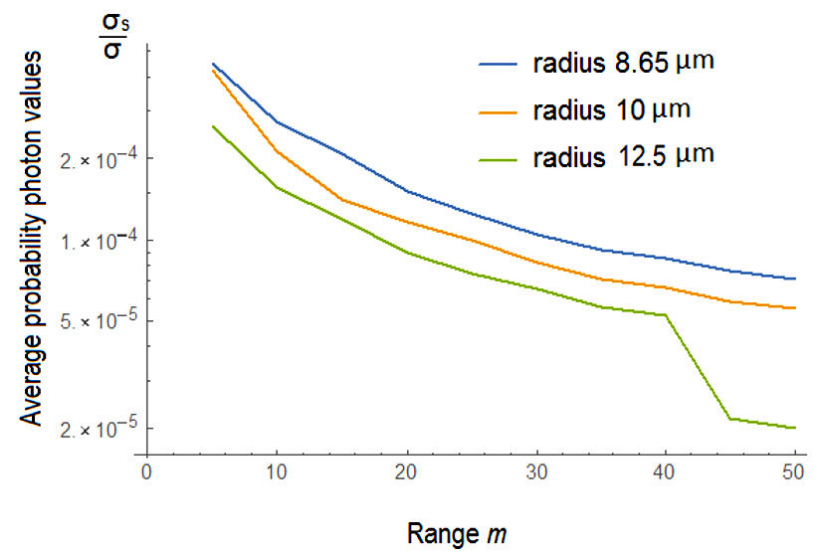

FIGURE 6. Profiles in the FOV. 


\begin{tabular}{|c|c|c|c|c|}
\hline \multicolumn{5}{|c|}{ TABLE I. Scatterer radius $(r=8.65 \mu \mathrm{m})$} \\
\hline Incident Photons & wavelength & Caught Photons & Transmitted Photons & Backscattering Photons \\
\hline average & $(\mathrm{nm})$ & average & average & average \\
\hline $5.5 \times 10^{5}$ & 532 & $4.430 \times 10^{-16}$ & 0.9710 & 0.00957 \\
\hline \multicolumn{5}{|c|}{ TABLE II. Scatterer radius $(r=10 \mu \mathrm{m})$} \\
\hline Incident Photons & wavelength & Caught Photons & Transmitted Photons & Backscattering Photons \\
\hline average & $(\mathrm{nm})$ & average & average & average \\
\hline $5.5 \times 10^{5}$ & 532 & $3.383 \times 10^{-18}$ & 0.9713 & 0.00925 \\
\hline \multicolumn{5}{|c|}{ TABLE III. Scatterer radius $(r=12.5 \mu \mathrm{m})$} \\
\hline Incident Photons & wavelength & Caught Photons & Transmitted Photons & Backscattering Photons \\
\hline average & $(\mathrm{nm})$ & average & average & average \\
\hline $5.5 \times 10^{5}$ & 532 & $3.439 \times 10^{-18}$ & 0.9742 & 0.00637 \\
\hline
\end{tabular}

\section{Validation of the model with an experimen- tal lidar signal}

The Monte Carlo-Mie model was performed to represent a distance profile of experimental data, provided by courtesy of THE FRENCH AEROSPACE LAB (ONERA). Referring to a backscattered Lidar signal from turbid media in clouds located at a minimum height of one thousands sixty meters with a thickness of one hundred meters.

For that testing, the Monte Carlo-Mie algorithm used the following parameters, to fit the experimental profile: one thickness of turbid medium $h=100 \mathrm{~m}$, an energy laser pulse of $50 \mathrm{~mJ}$, assuming just $5.5 \times 10^{5}$ incident photons. Number of scattering events 100 . A wavelength of $355 \mathrm{~nm}$. A photodetection gain of eight times, this gain is assigned due to the limitations in the computer equipment used in this work, the $5.5 \times 10^{5}$ photons is a minimal energy fraction equivalent in order to femtojoules. Because of this, the authors assigned that gain compensation. One aperture radius of telescope of $0.1 \mathrm{~m}$ which it is located for each corresponding variation distance at (-R 00$)$. A relative refractive index $n_{r}=1.9996$ from turbid media $[5,11,12,13]$. And a radius of the droplets water from low ionization $r=10 \mu \mathrm{m}$ [14].

The algorithm started, while varying the distance of photodetector from 10650 to $1147.5 \mathrm{~m}$ by steps of $7.5 \mathrm{~m}$. Simulating the light pulse step through turbid media and for each step was necessary to make a numerical fit (factor fit). The factor fit applied to the total backscatterig list was proposed as Trasmittance factor [15]. As it is shown in Fig. 7.

Considering that factor fit, the authors getting sucssesfully modeling the experimental profile from backscattering power. Figure 8 shows theoretical and experimental power backscattering.

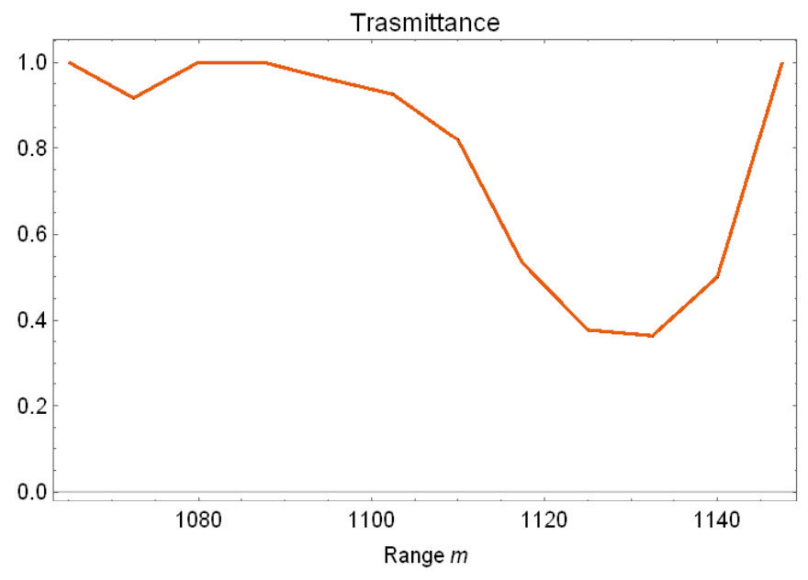

FIGURE 7. Trasmittance profile T(R).

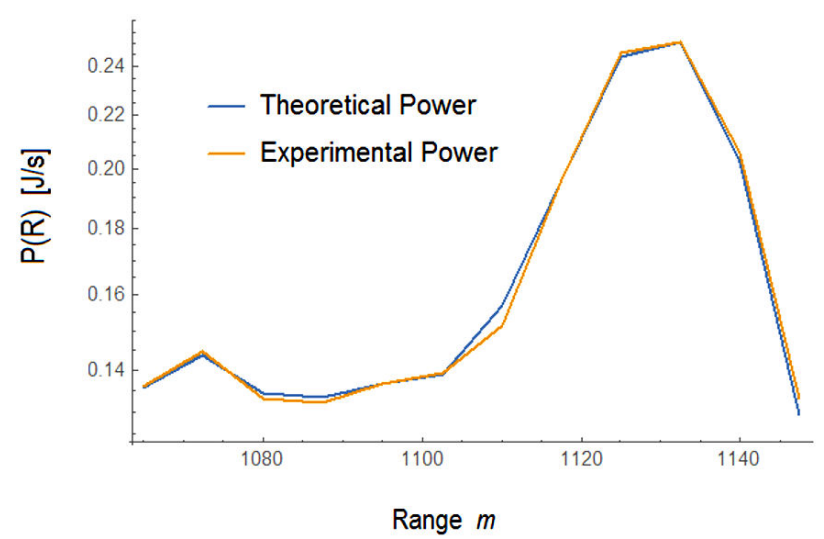

FIGURE 8. Theoretical and experimental backscattering power $\mathrm{P}(\mathrm{R})$, profiles. 


\section{Conclusions}

In scattering and quantification simulations of expanding as spherical harmonics waves, the authors are concluded:

Referring to Fig. 7, one factor fit is proposed, it was associate to this disturbance as a physical relation of the transmittance factor $T(R)$, that relates to the extinction coefficient at distance R. And it is applied at photodetector measurements, specific for each height over an interval from 1065 to $1147.5 \mathrm{~m}$. As a result of this, an experimental and theoretical signals lidar are closely fitted, as it is shown in Fig. 8. The success of this modeling is due to the turbid medium is being composed of spherical scatterers (water drops), with an average radius size of $10 \mu \mathrm{m}$ and the Monte Carlo-Mie model is based on vector bases that represents scattering spherical harmonic and consequently the authors proposed an empirical equation that represents the backscattering power as a function of the height. It is defined as [15].

$$
P(R, \lambda)=P_{0}\left\langle w C C(R, \lambda)_{\mathrm{FOV}}\right\rangle T(R)
$$

With $P_{0}$, is the power value of incident pulse $[\mathrm{J} / \mathrm{s}]$. $w C C(R, \lambda)_{\mathrm{FOV}}$, is the photon backscattering average [dimensionless].

$$
T(R)=\exp \left(-2 \int_{0}^{r} \alpha_{t, \lambda}(x) d x\right),
$$

is the transmittance factor [dimensionless].
1. L.R. Bissonnette et al., LIDAR multiple scattering from clouds, Appl. Phys. B 60 (1995) 355. https://doi.org/10. $1007 / \mathrm{BF} 01082271$

2. W. Zhensen, Y. Yi, and C. Lihong, Monte Carlo simulation for millimeter wave propagation and scattering in rain medium, International J. Infrared and Millimeter Waves 13 (1992) 7. https://doi.org/10.1007/BF01009622

3. P. F. Liaparinos, Light wavelength effects in submicrometer phosphor materials using Mie scattering and Monte Carlo simulation, Med. Phys. 40 (2013) 10, http://dx.doi.org/ $10.1118 / 1.4821089$

4. Y. Ma, W. Liu, Y. Cui, and X. Xiong, Multiple-scattering effects of atmosphere aerosols on light-transmission measurements, Opt. Rev. 24 (2017) 590. DOI: 10.1007/ S10043-017-0352-9

5. C. F. Bohren and D. R. Huffman, Absorption and Scattering of Light by Small Particles, (John Wiley \& Sons, 1983), pp. 57-63, 82-103, 111-112. ISBN 0-471-29340-7.

6. L. Tsang, J. Au Kong, K.-Hau Ding, Scattering of Electromagnetic Waves, (John Wiley \& Sons, 2000), pp. 32-41. ISBNs: 0-471-38799-1.

7. I. M. Sóbol, Método de Montecarlo, 2nd ed. (MIR Moscú, 1983), pp. 55-62.

8. S. Julius Adams, Electromagnetic Theory, (McGraw Hill, 1941), pp. 392-420.

9. B. Roysan, A. R. Cohen, P. H. Getto and P. R. Boyce, A Numerical Approach to the Computation of Light Propagation through
Turbid Media: Application to the Evaluation of Lighted Exit Signs, IEEE Transactions on industry applications, 29 (1993) 3.

10. E. Reynoso Lara, J. Davila Pintle, Y. E. Bravo-Garcia, A. Balbuena-Ortega, Numerical results of Monte Carlo code in lidar returns considering polarization of light and different phase functions, Opt. Pura Apl. 47 (2014) 177. http://dx.doi. org/10.7149/OPA.47.3.177

11. F. Fabregat-Santiago, N. S. Fierrols, G. García-Belmonte, J. Bisquert, and E. I. Morell, Estudio de los diferentes estados energéticos del agua del suelo en función de los fenómenos de relajación dieléctrica, Estudios de la Zona No Saturada del Suelo, (1999) 39-44. ISBN 84-699-1258-5.

12. Vaxasoftware, Tablas de propiedades Físicas, https: //www.vaxasoftware.com/doc_edu/fis/ inrefraccion.pdf

13. M. de la Vega Perez Gracia, Radar de subsuelo. Evaluación para aplicaciones en arqueología y en patrimonio históricoartístico, (2001), p. 206. ISBN: 846996884X.

14. T. Y. Nakajima, K. Suzuki, G. L. Stephens, Droplet Growth in Warm Water Clouds Observed by the A-Train. Part I: Sensitivity Analysis of the MODIS-Derived Cloud Droplet Sizes, Journal of the Atmospheric Sciences, 67 (2009) 1884-1896, DOI: $10.1175 / 2009$ JAS3280.1

15. C. Weitkamp, Lidar Range-Resolved Optical Remote Sensing of the Atmosphere, (Springer series in Optical Science, 2005), pp. 6-11. ISBN 0-387-40075-3. 